\title{
Addition of NMDA-receptor antagonist MK801 during oxygen/glucose \\ deprivation moderately attenuates the up-regulation of glucose uptake after subsequent reoxygenation in brain endothelial cells
}

Winfried Neuhaus $^{\mathrm{a}, \mathrm{b}, *}$, Malgorzata Burek ${ }^{\mathrm{a}}$, Cholpon S. Djuzenova ${ }^{\mathrm{c}}$, Serge Thal ${ }^{\mathrm{d}}$, Hermann Koepsell ${ }^{\mathrm{e}}$, Norbert Roewer ${ }^{\mathrm{a}}$, Carola Y. Förster ${ }^{\mathrm{a},{ }^{*}}$

${ }^{\mathrm{a} D e p a r t m e n t}$ of Anaesthesia and Critical Care, University of Wuerzburg, Overdürrbacherstrasse 6, 97080 Wuerzburg, Germany

${ }^{\mathrm{b}}$ Department of Medicinal Chemistry, University of Vienna, Althanstrasse 14, A-1090 Vienna, Austria

${ }^{\mathrm{c}}$ Department of Radiation Oncology, University of Wuerzburg, Josef-Schneider-Strasse 11, 97080 Wuerzburg, Germany

${ }^{\mathrm{d}}$ Department of Anesthesiology, Johannes Gutenberg University Mainz, Langenbeckstr. 1, 55131 Mainz, Germany

${ }^{\mathrm{e}}$ Department of Anatomy and Cell Biology, University of Wuerzburg, Koellikerstraße 6, D97070 Wuerzburg, Germany

*corresponding authors:

Prof. Dr. Carola Y. Förster, Department of Anaesthesia and Critical Care, University of Wuerzburg, Overdürrbacherstrasse 6, 97080 Wuerzburg, Germany, Foerster_C@klinik.uniwuerburg.de

Dr. Winfried Neuhaus, Department of Medicinal Chemistry, University of Vienna, Althanstrasse 14, A-1090 Vienna, Austria, winfried.neuhaus@univie.ac.at 


\section{Abstract}

During stroke the blood-brain barrier $(\mathrm{BBB})$ is damaged which can result in vasogenic brain edema and inflammation. The reduced blood supply leads to decreased delivery of oxygen and glucose to affected areas of the brain. Oxygen and glucose deprivation (OGD) can cause upregulation of glucose uptake of brain endothelial cells. In this letter, we investigated the influence of MK801, a non-competitive inhibitor of the NMDA-receptor, on the regulation of the glucose uptake and of the main glucose transporters glut1 and sglt 1 in murine BBB cell line cerebEND during OGD. mRNA expression of glut1 was upregulated 68.7-fold after six hours OGD, which was significantly reduced by $10 \mu \mathrm{M}$ MK801 to 28.9-fold. Sglt1 mRNA expression decreased during OGD which was further reduced by MK801. Glucose uptake was significantly increased up to $907 \%$ after six hours OGD and was still higher (210\%) after the 20 hours reoxygenation phase compared to normoxia. Ten $\mu \mathrm{M}$ MK801 during OGD was able to reduce upregulated glucose uptake after OGD and reoxygenation significantly. Presence of several NMDAR subunits was proven on the mRNA level in cerebEND cells. Furthermore, it was shown that NMDAR subunit NR1 was upregulated during OGD and that this was inhibitable by MK801. In conclusion, the addition of MK801 during the OGD phase reduced significantly the glucose uptake after the subsequent reoxygenation phase in brain endothelial cells.

Key words: blood-brain barrier, MK801, NMDAR, stroke, glut1, sglt1 


\section{Introduction}

The main fuel for the brain is glucose. Glucose is mainly transported by the highly glycosylated $55 \mathrm{kDa}$ form of the sodium independent, facilitative glucose transporter glut1 across the blood-brain barrier (BBB) into the CNS [22]. During stroke the reduction of blood supply results in a decrease of oxygen and glucose in local areas of the brain. Ischemia followed by reperfusion leads to BBB disruption and the increased cerebrovascular permeability contributes to vasogenic brain edema and inflammation [1,9] which can influence the stroke outcome significantly. Glucose transporters are upregulated by hypoxia as well as aglycemia in several tissues and cell types [13]. This upregulation is potentiated when oxygen and glucose deprivation (OGD) is applied in combination at the same time. The therapeutical approaches for stroke are limited. Recently, Espinoza-Rojo et al. [6] suggested to investigate glucose transporter as novel targets for the treatment of stroke. Since it is known that glucose transporters transport water together with glucose in order to compensate for the osmotic pressure $[14,23,24]$, it was hypothesized that this co-transport of water may contribute to the formation of edema during stroke. Thus, it could be worthy to elucidate the underlying mechanisms leading to the upregulation of glut1 at the BBB. Four main incidents are known to cause glut1-upregulation: 1) glutamate excitotoxicity 2) hypoxia 3) aglycemia and 4) mitochondrial damage. Glutamate excitotoxicity and OGD can result in the formation of reactive oxygen species (ROS). ROS can cause mitochondrial damage [6]. In this context, it was recently reported that NMDAR inhibitor MK801 was able to block the upregulation of ROS formation and the following disruption of the barrier's integrity after hypoxia or glutamate stimuli in in vitro BBB models $[11,12]$. Thus, the first aim of the presented work was to investigate our hypothesis that MK801 can reduce the upregulation of glut1 during OGD in brain endothelial cells and to elucidate the contribution of this inhibition onto the total glucose uptake. Recently, presence and upregulation of sodium coupled glucose 
transporter 1 (sglt1) during OGD was reported in bovine brain endothelial cells [23]. Furthermore, functionality of sglts in BBB models was shown in vitro and an important role for sglts in vivo in edema formation during stroke was suggested [15,23]. However, sglt functionality at the BBB under physiological conditions is under doubt [25]. Consequently, we included sglt 1 in our investigations and tested presence and regulation of sglt 1 in our BBB model based on murine cell line cerebEND [20]. For our BBB in vitro model we have chosen to use cerebEND cells since they possess key BBB properties and markers, were tighter and exhibit an increased sensitivity to stimuli in comparison to other BBB in vitro models based on cell lines $[18,20]$.

\section{Material and Methods}

Murine brain endothelial cell line cerebEND was generated from isolated brain microvascular endothelial cells from mouse cerebellum by Silwedel and Foerster [20]. cerebENDs were cultured in DMEM medium supplemented with $10 \%$ FCS and $1 \%$ penicillin/streptomycin onto gelatine coated cell culture tissue flasks and were subcultivated by trypsination in a ratio of $1: 3$ once a week. Cells were maintained in an incubator at $37^{\circ} \mathrm{C}, 95 \%$ humidity and a $5 \%$ $\mathrm{CO}_{2} / 95 \%$ air atmosphere. For mRNA as well as glucose uptake studies cerebENDs were seeded at a density of 20,000 cells $/ \mathrm{cm}^{2}$ in gelatine-coated 6-well plates and were grown to confluence within 5 days of culture. On day 5 after seeding serum amount of the growth medium was reduced to $1 \%$ FCS-SS in DMEM to enhance differentiation of the cells. On the next day oxygen/glucose deprivation (OGD) was applied on cerebEND cells in an InVivo 2 hypoxia work station (Ruskinn) with $0.5 \% \mathrm{O}_{2}, 5 \% \mathrm{CO}_{2}$, saturated humidity atmosphere and $37^{\circ} \mathrm{C}$ for 6 hours [9]. After the OGD phase cells were immediately used for mRNA analysis or glucose uptake studies or a subsequent 20 hour reoxygenation phase was accomplished. MK801 was only added during the 6 h OGD phase to the growth media. 
For mRNA analysis cells were lysed and RNA was isolated using the RNeasy Mini Kit (Quiagen) according to the manufacturer instructions. mRNA for qPCR analysis of glut1 and NMDAR subunits NR1, NR2A, NR2B, N2C, NR2D, NR3A and NR3B was reverse transcribed into cDNA by means of the Verso-kit (ThermoScientific) using oligodT primers according to the manufacturer's instruction. For cDNA synthesis of low abundant target sglt1 the high capacity cDNA-kit from Applied Biosystems was applied followed by a cDNA preamplification step before $\mathrm{qPCR}$ was conducted. $0.85 \mu \mathrm{g}$ total RNA per sample were transcribed to $20 \mu \mathrm{L}$ cDNA. cDNA for 18 SrRNA and sglt1 was then preamplified according to the protocol provided by Applied Biosystems. qPCR analysis were performed using either Taqman ${ }^{\circledR}$ probes (Applied Biosystems) for glut-1 (Mm01192270_m1), sglt1 (Mn01218039_m1) and 18SrRNA (endogenous control, EUK18SrRNA, 4352930-0810022) or primers from MWG Eurofins with SYBRGreen for NR1 (fw:CTCTAGCCAGGTCTACGCTATCC, rev:GACGGGGATTCTGTAGAAGCCA; specific for all 3 transcript variants, accession numbers: NM_001177657.1; NM_001177656.1; NM_008169.2), NR2A (fw:ACATCCACGTTCTTCCAGTTTGG, rev:GACATGCCAGTCATAGTCCTGC; NM_008170.2), NR2B (fw:CCAGAGTGAGAGATGGGATTGC, rev:TGGGCTCAGGGATGAAACTGT; NM_008171.3), $\quad$ NR2C (fw:GGGCTTCTGCATCGACATCC, rev:ATCATACCATTCCACACACCACG; NM_010350.2), NR3A (fw:ATCCTCAAGCGCATCGGACA, rev:CGACTCTGGCTCATCCCTCTG; NM_001033351.1), NR3B (fw:GGCCGTGACCAGCTTCAGTA, rev:CAATGGGTGAGGCTGTATCTCG; NM_130455.2) and 18SrRNA (fw:ATGGTTCCTTTGGTCGCTCG，rev:GAGCTCACCGGGTTGGTTTT; NR_003278.2) in $25 \mu \mathrm{L}$ total volume per well. All qPCR analysis were conducted by means of a 7300 RealTime PCR System (Applied Biosystems). Each sample was analyzed as triplicate. Relative mRNA abundances to 18 SrRNA were calculated by the $\mathrm{dCt}$ method using following formula: 
$2^{\text {(Ct of } 18 \mathrm{~S} \text { rRNA-Ct of gene of interest) }}$, where $\mathrm{Ct}$ is the threshold cycle value. For the qualitative proof of the presence of NMDAR subunits cDNA samples amplification products were separated on a $2 \%$ agarose gel in TAE-buffer with a peqGOLD 50 bp DNA-ladder as marker at $90 \mathrm{~V}$ for half an hour.

For glucose uptake studies cell layers were washed with prewarmed PBS twice and were preincubated with PBS (with $\mathrm{Ca}^{2+} / \mathrm{Mg}^{2+}$ ) containing glucose transporter inhibitors $(10 \mu \mathrm{M}$ phloretin for glut-1 blockade, $20 \mathrm{mM}$ galactose for sglt 1 blockade) at $37^{\circ} \mathrm{C}$ for 20 minutes. After preincubation $50 \mu \mathrm{M}\left[{ }^{3} \mathrm{H}\right]$-glucose $(1 \mathrm{mCi} / \mathrm{mL}, 50-60 \mathrm{Ci} / \mathrm{mmol}, 0113 \mathrm{~A}, \mathrm{ARC})$ supplemented with inhibitors were added. After 15 minutes at $37^{\circ} \mathrm{C}$ cell layers were washed with ice-cold PBS for three times. Cells were then lysed with $1 \%$ Triton-X 100 solution and lysates were added to 3097 Lumasafe plus solution (Lumac-lsc B.V.) to measure radioactivity by a beta-counter LS 6500 (Beckman). Each sample was determined as triplicate and at least three samples were collected for each condition $(n=3)$. Resulted dpm values were normalized to according sample's protein content (Pierce BCA assay, Thermo Scientific) and were related to appropriate normoxic controls which were set to $100 \%$ and to radioactivity of each applied stock solution. For cell viability tests (EZ4U, Biomedica) cerebENDs were seeded in gelatinecoated 96-well microplates and were treated under the same OGD conditions as explained above. Cell viability tests were carried out according to the manufacturer's instruction. Details of the methods are described in the electronic supplementary file available on the Journal's website. 


\section{Results and Discussion}

The main glucose transporter in brain capillary endothelial cells is glut1 [22]. Thus, the regulation of glut1 in murine cerebEND cells was investigated during normoxic, hypoxic and OGD conditions. Cell viability measurements revealed no cytotoxic effect independent of each treatment modality on cerebEND cells (data not shown). Results shown in figure 1 clearly showed the upregulation of glut1 during the six hours under hypoxic and OGD conditions compared to the normoxic experiment. For example, glut1 was upregulated after six hours during hypoxia 15.4-fold $(\mathrm{p}<0.001)$ and 68.7 -fold $(\mathrm{p}<0.001)$ during OGD. This ranking order of differential answers to different hypoxia/aglycemia stimuli was completely in concordance to results of Abbruscato and Davis [1] who showed that the permeability of a blood-brain barrier in vitro model increased stepwise dependent on application of aglycemia, hypoxia and OGD. Addition of MK801 decreased glut1 mRNA expression during hypoxia and OGD. For example, presence of $10 \mu \mathrm{M}$ MK801 during OGD reduced significantly glut1 upregulation from 68.7 to 28.9-fold $(\mathrm{p}<0.05)$. During hypoxia MK801 decreased glut1 expression in a concentration dependent manner, whereby addition of $1 \mu \mathrm{M}$ MK801 yielded in the most distinct, but not significant effect and reduced glut1 expression from 15.4 to 11.4fold compared to normoxia. Addition of MK801 during OGD revealed no significant differences after subsequent reoxygenation in comparison to the appropriate hypoxia and OGD samples.

Since strongest effects on glut1 regulation were found after OGD, further investigations were done under OGD conditions. Recently, presence and functional relevance of a second glucose transporter at the $\mathrm{BBB}$, called sglt1, was reported [4,15,23]. Results shown in figure 2 revealed that mRNA of sglt1 was significantly downregulated during six hours of OGD (0.57fold, $\mathrm{p}<0.001)$ compared to according normoxia samples. Addition of $1 \mu \mathrm{M}$ MK801 reduced sglt1 expression to $49 \%$, which was further decreased by $10 \mu \mathrm{M}$ MK801 to $45 \%$ after OGD in 
comparison to normoxia $(\mathrm{p}<0.001$; but not statistically significant compared to OGD). After the additional reoxygenation sglt1 expression of the OGD treated cells approximated to the corresponding normoxia controls. Only treatment during OGD with $10 \mu \mathrm{M}$ MK801 revealed significantly lower sglt1 expression than normoxic controls $(\mathrm{p}<0.05)$.

As next step we investigated if murine blood-brain barrier cell line cerebEND expresses NMDA receptor subunits and whether their expression could also be regulated by addition of their blocker MK801. First, presence of NMDAR subunit transcripts of NR1, NR2A, NR2B, NR2C, NR3A and NR3B was proven by PCR (figure 3A). A conventional, functionally active NMDAR has to possess NR1 subunits. Furthermore, Reijerkerk et al. [19] confirmed a relevant role of the NR1 subunit at the BBB in vitro during monocyte migration. Consequently, we analyzed whether mRNA expression of NR1 had been regulated during OGD and if this was influenced by NMDAR blocker MK801. Results depicted in figure 3B revealed a distinct upregulation of NR1 during OGD and that this was counterregulated by NMDAR inhibitor MK801. In detail, NR1 mRNA was upregulated after six hours OGD up to 2.9-fold in comparison to normoxia which persisted at a level of 3.1-fold after the additional 20 hours of reoxygenation (both $\mathrm{p}<0.05$ ). MK801 was able to decrease NR1 upregulation during OGD and reduced NR1 expression after additional 20 hours reoxygenation to 1.66- (10 $\mu \mathrm{M}$ MK801, $\mathrm{p}>0.05)$ and 0.68-fold $(100 \mu \mathrm{M}$ MK801, $\mathrm{p}<0.05)$.

Our presented findings here showed significant upregulation of glucose transporter glut 1 and NR1 after OGD and a reduction of their upregulation by addition of MK801. However, no complete blockade of the upregulations was achieved by MK801. Thus, in order to investigate the functional relevance of regulated mRNA expression of glucose transporters glut1 and sglt1, glucose uptake studies were accomplished. It was decided to add $10 \mu \mathrm{M}$ MK801 during OGD since this concentration of MK801 exhibited the strongest inhibitory effects on glut1 and sglt 1 mRNA expression after 6 hours OGD. Figure 4 shows that glucose uptake was significantly increased up to $907 \pm 69 \%(\mathrm{p}<0.05)$ directly after six hours of OGD treatment. 
After a following 20 hours reoxygenation phase glucose uptake was still significantly higher $(210 \pm 12 \%, \mathrm{p}<0.05)$ than the normoxia control. Addition of $10 \mu \mathrm{M}$ MK801 reduced glucose uptake to $845 \pm 20 \%$ measured directly after OGD (statistically not significant) and significantly decreased it to $163 \pm 9 \%$ after the additional reoxygenation phase $(\mathrm{p}<0.05)$. To elucidate the roles of glut 1 and sglt 1 in the glucose uptake rates phloretin, a glut 1 inhibitor [7], and galactose, a sglt1 inhibitor [3], were added during the uptake studies. Directly after OGD $10 \mu \mathrm{M}$ phloretin significantly reduced the uptake of glucose to $170 \pm 4 \%(\mathrm{p}<0.05)$, whereas supplementation of $20 \mathrm{mM}$ galactose revealed a moderate, but also significant decrease to $657 \pm 14 \%(\mathrm{p}<0.05)$. Interestingly, phloretin still decreased glucose uptake after the additional reoxygenation phase from $210 \pm 13 \%$ to $57 \pm 2 \%(\mathrm{p}<0.05)$, whereas galactose only reduced the glucose uptake to $174 \pm 12 \%$. These results indicated that both transporters glut1 and sglt1 contributed to the increased glucose uptake during OGD. This was in concordance to first studies of Matsuoka et al. [15] showing functional presence of sglts at the $\mathrm{BBB}$ in vitro. In order to assign the reducing effects of MK801 on glucose uptake during OGD specifically to single glucose transporters, their regulation on the protein level has to be studied and known non-genomic mechanisms of the regulation of the transporter's functionality have to be included into the considerations [10]. These regulatory processes at the protein level are probably responsible for possible discrepancies between our observations on the mRNA and on the functional level. Western blots showed the up-regulation of glut1 and sglt1 in cerebENDs after OGD and reoxygenation on the protein level (see supplementary figure 1) supporting our glucose uptake results and confirming data from the literature that glucose transporters are posttranscriptionally regulated by processes which can control mRNA stability, transporter concentrations at the luminal surface and their affinities to glucose [6,21]. Furthermore, presence and function of other glucose transporters at the BBB should not be neglected. For example, Enerson and Drewes [5] reported presence of mRNA of the glucose transporter sglt 2 in rat brain capillaries. However, in our present report we 
show that glut1 expression is affected by different glucose/oxygen conditions in the murine blood-brain barrier cell line cerebEND and that the expression of glut 1 can be influenced by NMDAR blocker MK801. Furthermore, to our best knowledge we are the first who report the presence of several NMDAR subunits in cerebEND cells and the significant upregulation of NR1 during OGD and that this can be blocked by NMDAR inhibitor MK801. Moreover, we were able to prove the hypothesis that MK801 is able to decrease the upregulation of glucose transporter glut1 during OGD and to reduce the elevated glucose uptake after OGD. However, the direct link between the regulation of glucose transporters and NMDAR is not fully resolved. Hypoxia leads to an increase of the intracellular Ca-level by influx of extracellular Ca-ions as well as by a Ca-release from intracellular stores at the BBB in vitro, and Casignalling under hypoxia plays a crucial role for the formation of ROS [12]. Then, ROS damage membranes of mitochondria impairing oxidative phosphorylation which result in the upregulation of glucose transporters [6]. Under hypoxia astrocytes play an important role in these processes since they release glutamate to e.g. modulate NMDAR functionality or VEGF to stimulate glut1 expression via HIF-1 and HRE elements [6,12]. Interestingly, in the present work we have observed effects by MK801 in our BBB in vitro model without using astrocytes indicating the presence of alternative pathways. Recently, deleterious effects of homocysteine onto the BBB were reported to be mediated by NMDAR [2]. Furthermore, we and others were able to show that MK801 alone can affect the proteome and the intracellular Ca-level of BBB models $[16,17]$. Consequently, it should be taken into consideration that currently unknown serum components may act as NMDAR agonists or that MK801 can affect the intracellular Ca-level without prior NMDAR stimulation which can lead to decreased ROS formation and subsequent lower glucose transporter functionality.

In conjunction with the postulated co-transport of water and glucose molecules [14], the treatment with NMDAR-antagonists may result in reduced edema formation after stroke. In this context, further studies concerning NMDAR functionality and underlying signalling 
pathways involved in the treatment with NMDAR antagonists at the $\mathrm{BBB}$ are required in order to identify suitable downstream targets for future stroke therapies.

\section{Acknowledgements}

The research leading to these results has received funding from the European Union Seventh Framework Programme (FP7/2007-2013) under grant agreement $\mathrm{n}^{\circ}$ HEALTH-F2-2009241778 and from the Deutsche Forschungsgemeinschaft DFG under grant number FO 315/41. We thank Bianca Schneiker ${ }^{\mathrm{a}}$, Elisabeth Wilken ${ }^{\mathrm{a}}$, Anja Neuhoff ${ }^{\mathrm{a}}$ and Ursula Roth ${ }^{\mathrm{e}}$ for their for excellent technical assistance. 


\section{References}

[1] T.J. Abbrucato, T. Davis, Combination of Hypoxia/Aglycemia Compromises In Vitro Blood-Brain Barrier Integrity, JPET 289 (1999) 668-675.

[2] R.S. Beard, J.J. Reynolds, S.E. Bearden, Hyperhomocysteinemia increases permeability of the blood-brain barrier by NMDA receptor-dependent regulation of adherens and tight junctions, Blood. 118(2011) 2007-2014.

[3] A. Diez-Sampedro, E.M. Wright, B.A. Hirayama, Residue 457 controls sugar binding and transport in the $\mathrm{Na}(+) /$ glucose cotransporter, JBC 276 (2001) 49188-49194.

[4] K. Elfeber, A. Köhler, M. Lutzenburg, C. Osswald, H.J. Galla, O.W. Witte, H. Koepsell, Localization of the Na-D-glucose cotransporter SGLT1 in the blood-brain barrier. Histochem Cell Biol 121 (2004) 201-207.

[5] B.E. Enerson, L.R. Drewes, The rat blood-brain barrier transcriptome, J. Cereb. Blood Flow Metab. 26 (2006) 959-973.

[6] M. Espinoza-Rojo, K.I. Iturralde-Rodríguez, M.E. Chánez-Cárdenas, M.E. RuizTachiquín, P. Aguilera, Glucose transporters regulation on ischemic brain: possible role as therapeutic target, Central Nervous System Agents in Medicinal Chemistry 10 (2010) 317325.

[7] T. Kasahara, M. Maeda, E. Boles, M. Kasahara, Identification of a key residue determining substrate affinity in the human glucose transporter GLUT1, Biochim. Biophys. Acta. 1788 (2009) 1051-1055.

[8] J.Y. Khan, R.A. Rajakumar, R. MCKnight, U.P. Devaskar, S.U. Devaskar, Developmental regulation of genes mediating murine brain glucose uptake, Am J Physiol Regulatory Integrative Comp Physiol 276 (1999) 892-900.

[9] C. Kleinschnitz, K. Blecharz, T. Kahles, T. Schwarz, P. Kraft, K. Göbel, S.G. Meuth, M. Burek, T. Thum, G. Stoll, C. Förster, Glucocorticoid insensitivity at the hypoxic blood-brain barrier can be reversed by inhibition of the proteasome, Stroke 42 (2011) 1081-1089. 
[10] M. Kroiss, M. Leyerer, V. Gorboulev, T. Kühlkamp, H. Kipp, H. Koepsell, Transporter regulator RS1 (RSC1A1) coats the trans-Golgi network and migrates into the nucleus, Am J Physiol Renal Physiol. 291(2006) F1201-F1212.

[11] C.R.W. Kuhlmann, M. Gerigk, B. Bender, D. Closhen, V. Lessmann, H.J. Luhmann, Fluvastatin prevents glutamate-induced blood-brain-barrier disruption in vitro. Life Sci. 82 (2008) 1281-1287.

[12] C.R. Kuhlmann, C.M. Zehendner, M. Gerigk, D. Closhen, B. Bender, P. Friedl, H.J. Luhmann, MK801 blocks hypoxic blood-brain-barrier disruption and leukocyte adhesion, Neurosci Lett. 449 (2009) 168-172.

[13] J.D. Loike, L. Cao, J. Brett, S. Ogawa, S.C. Silverstein, D. Stern, Hypoxia induces glucose transporter expression in endothelial cells, Am J Physiol. 263 (1992) C326-C333.

[14] N. MacAulay, T. Zeuthen, Water transport between CNS compartments: contributions of aquaporins and cotransporters, Neuroscience 168 (2010) 941-956.

[15] T. Matsuoka, T. Nishizaki, G.E. Kisby, Na+-dependent and phlorizin-inhibitable transport of glucose and cycasin in brain endothelial cells, J Neurochem. 70 (1998) 772-777. [16] A. Minagar, J.S. Alexander, R.E. Kelley, M. Harper, M.H. Jennings, Proteomic analysis of human cerebral endothelial cells activated by glutamate/MK-801: significance in ischemic stroke injury, J. Mol. Neurosci. 38 (2009) 182-192.

[17] W. Neuhaus, M. Freidl, P. Szkokan, M. Berger, M. Wirth, J. Winkler, F. Gabor, C. Pifl, C.R. Noe, Effects of NMDA receptor modulators on a blood-brain barrier in vitro model, Brain Res. 1394 (2011) 49-61.

[18] B. Poller, H. Gutmann, S. Krähenbühl, B. Weksler, I. Romero, P.O. Couraud, G. Tuffin, J. Drewe, J. Huwyler, The human brain endothelial cell line hCMEC/D3 as a human bloodbrain barrier model for drug transport studies, J. Neurochem. 107 (2008) 1358-68. 
[19] A. Reijerkerk, G. Kooij, S.M. van der Pol, T. Leyen, K. Lakeman, B. van Het Hof, D. Vivien, H.E. de Vries, The NR1 subunit of NMDA receptor regulates monocyte transmigration through the brain endothelial cell barrier, J Neurochem. 113 (2010) 447-453.

[20] C. Silwedel, C. Förster, Differential susceptibility of cerebral and cerebellar murine brain microvascular endothelial cells to loss of barrier properties in response to inflammatory stimuli, J Neuroimmunol. 179 (2006) 37-45.

[21] I.A. Simpson, N.M. Appel, M. Hokari, J. Oki, G.D. Holman, F. Maher, E.M. KoehlerStec, S.J. Vannucci, Q.R. Smith, Blood-Brain Barrier Glucose Transporter: Effects of Hypoand Hyperglycemia Revisited, J. Neurochem. 72 (1999) 238-247.

[22] S.J. Vanucci, F. Maher, I.A. Simpson, Glucose transporter proteins in brain: delivery of glucose to neurons and glia, Glia 21 (1997) 2-21.

[23] S. Vemula, K.E. Roder, T. Yang, G.J. Bhat, T.J. Thekkumkara, T.J. Abbruscato, A functional role for sodium-dependent glucose transport across the blood-brain barrier during oxygen glucose deprivation, JPET 328 (2009) 487-495.

[24] E.M. Wright, E. Turk, The sodium/glucose cotransport family SLC5, Pflugers Arch. 447 (2004) 510-518.

[25] A.S. Yu, B.A. Hirayama, G. Timbol, J. Liu, E. Basarah, V. Kepe, N. Satyamurthy, S.-C. Huang, E.M. Wright, J.R. Barrio, Functional expression of SGLTs in rat brain, Am. J. Physiol. Cell Physiol. 299 (2010) C1277-1284. 


\section{Figure legends}

Figure 1: Concentration dependent regulation of glut1 mRNA in cerebEND cells by MK801 during six hours hypoxia and OGD and subsequent 20 hours reoxygenation (RO). Data are presented as means \pm SEM $(n=5-9)$. Statistically significant differences are marked by $*$ ( $\mathrm{p}<0.05$, two-sided t-test with same variances) and $* *(\mathrm{p}<0.001$, two-sided $\mathrm{t}$-test with same variances) in comparison to normoxia and \# ( $\mathrm{p}<0.05$, two-sided t-test with same variances) in comparison to OGD conditions.

Figure 2: Concentration dependent regulation of sglt1 mRNA in cerebEND cells by MK801 during six hours OGD and a subsequent 20 hours reoxygenation (RO) phase. Data are presented as means \pm SEM ( $n=6-9)$. Statistically significant differences are marked by * ( $\mathrm{p}<0.05$, two-sided t-test with same variances) and $* *(\mathrm{p}<0.001$, two-sided $\mathrm{t}$-test with same variances) in comparison to normoxia.

Figure 3: Presence of NMDAR-subunits (NR1: 106 bp, NR2A: 87 bp, NR2B: 81 bp, NR2C: 120 bp, NR3A: 132 bp, NR3B: 119 bp) in cerebEND cells (A) and the concentration dependent regulation of subunit NR1 by MK801 during six hours OGD and additional 20h reoxygenation (RO) phase (B). Data are presented as means \pm SEM ( $n=5-9)$. Statistically significant differences are marked by $*(\mathrm{p}<0.05$, two-sided t-test with same variances) in comparison to normoxia and by \# $(\mathrm{p}<0.05$, two-sided t-test with same variances $)$ in comparison to OGD conditions.

Figure 4: Increased $\left[{ }^{3} \mathrm{H}\right]$-glucose uptake of cerebEND cells after six hours OGD and subsequent 20 hours reoxygenation (RO) is blocked by $10 \mu \mathrm{M}$ phloretin (PT, glut1-inhibitor) and $20 \mathrm{mM}$ galactose (GAL, sglt1-inhbitor). Addition of $10 \mu \mathrm{M}$ MK801 during 6h OGD 
Original publication in: Neuhaus et al., Neuroscience Letters 506 (2012) 44- 49;

http://dx.doi.org/10.1016/j.neulet.2011.10.045

phase reduces the glucose uptake after the reoxgenation phase significantly. Data are presented as means \pm SEM $(n=3)$. Statistically significant differences are marked by * ( $\mathrm{p}<0.01$, two-sided t-test with same variances) in comparison to normoxia and by \# $(\mathrm{p}<0.05$, two-sided t-test with same variances) in comparison to OGD conditions. 
Figure 1

6h hypoxia

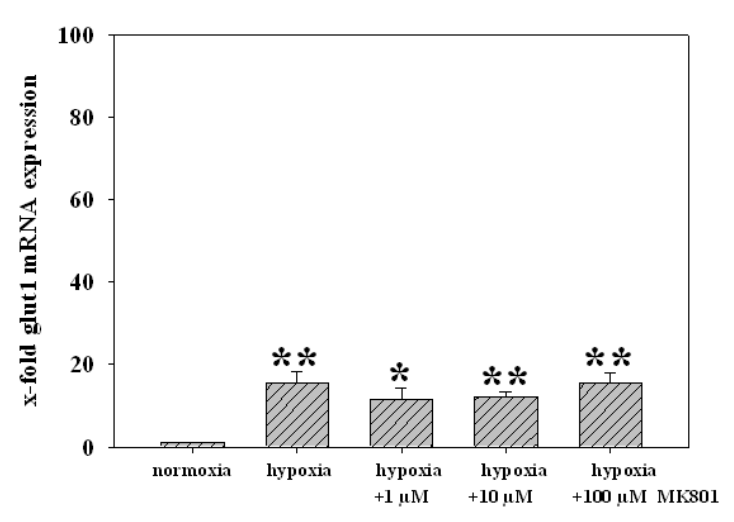

6h hypoxia $+20 h$ RO

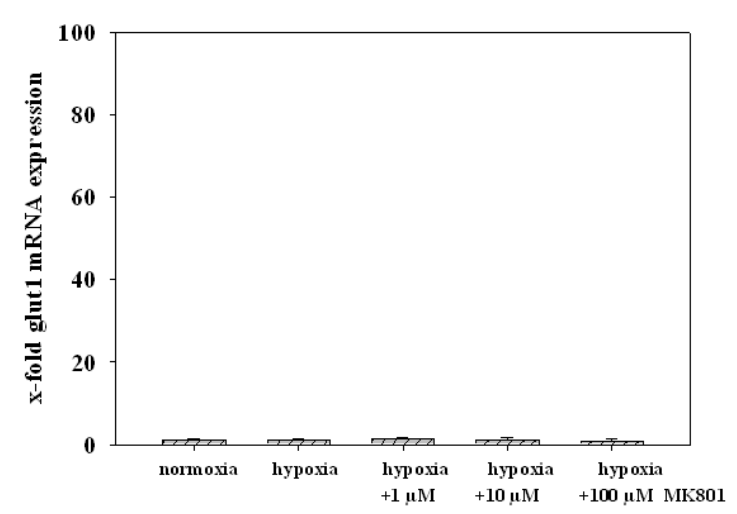

6h OGD

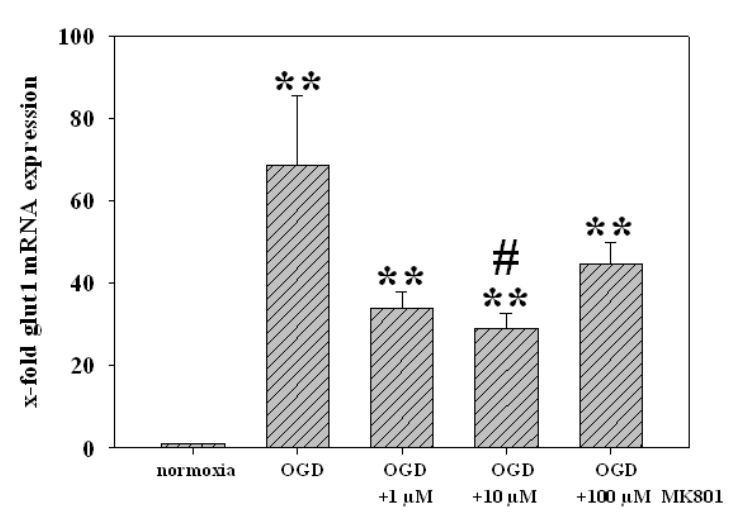

6h OGD + 20h RO

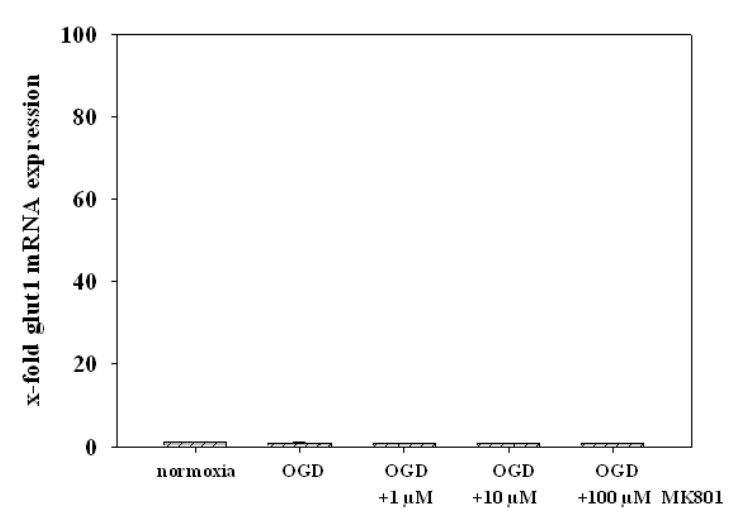




\section{Figure 2:}

\section{6h OGD}

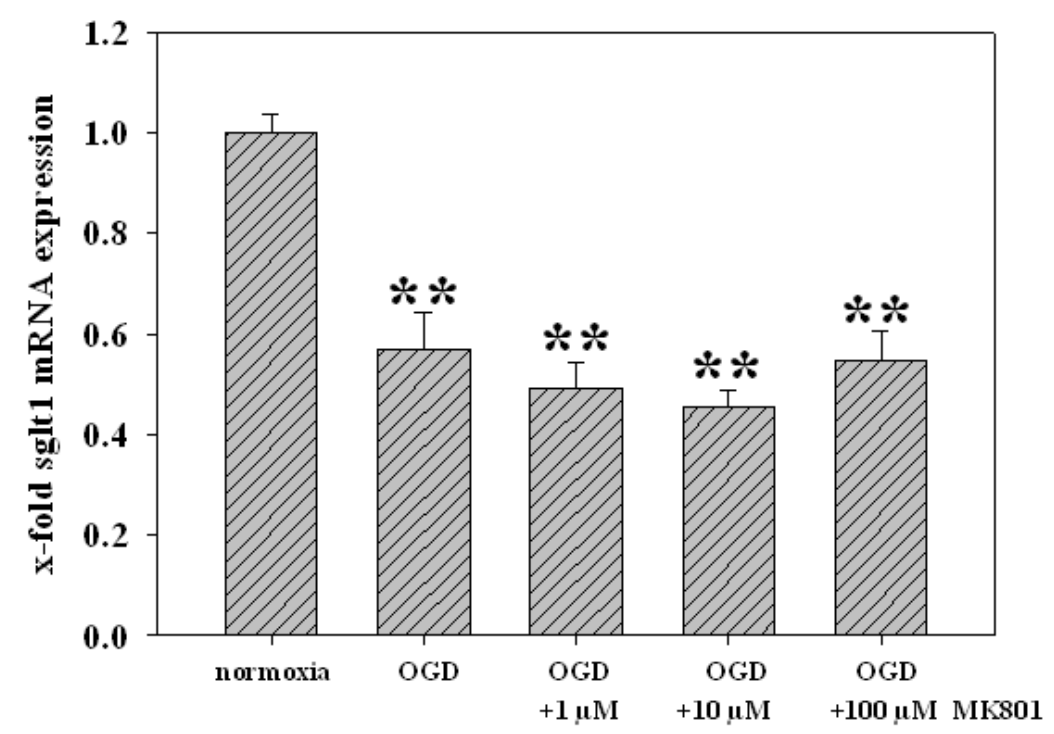

6h OGD + 20h RO

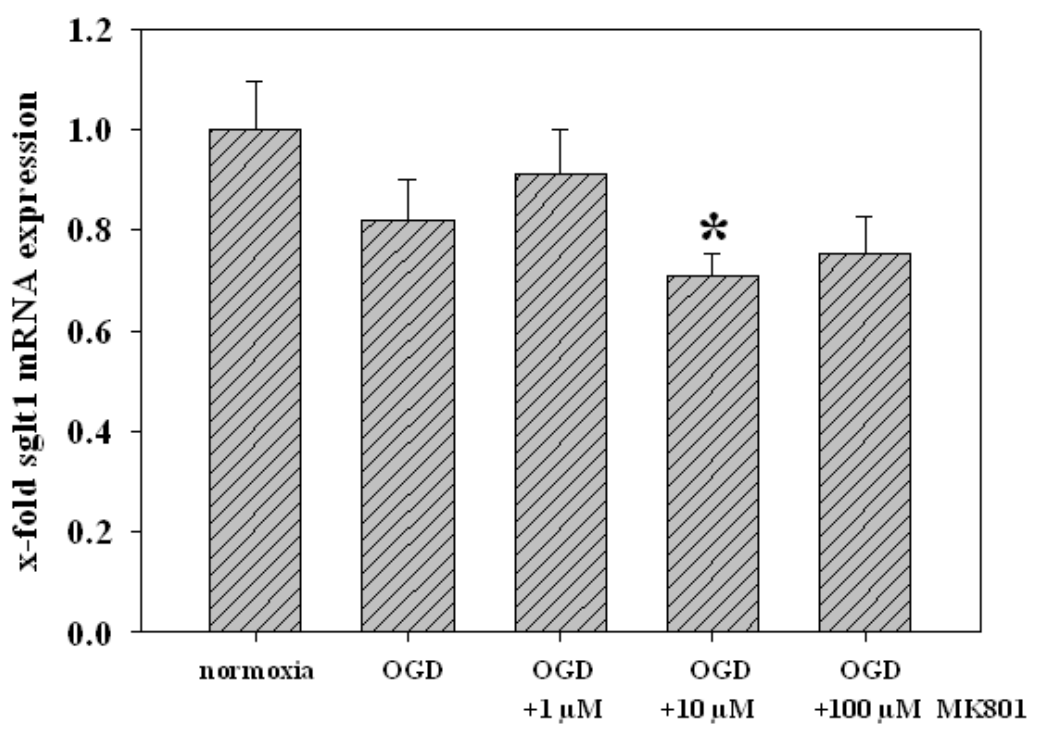




\section{Figure 3:}
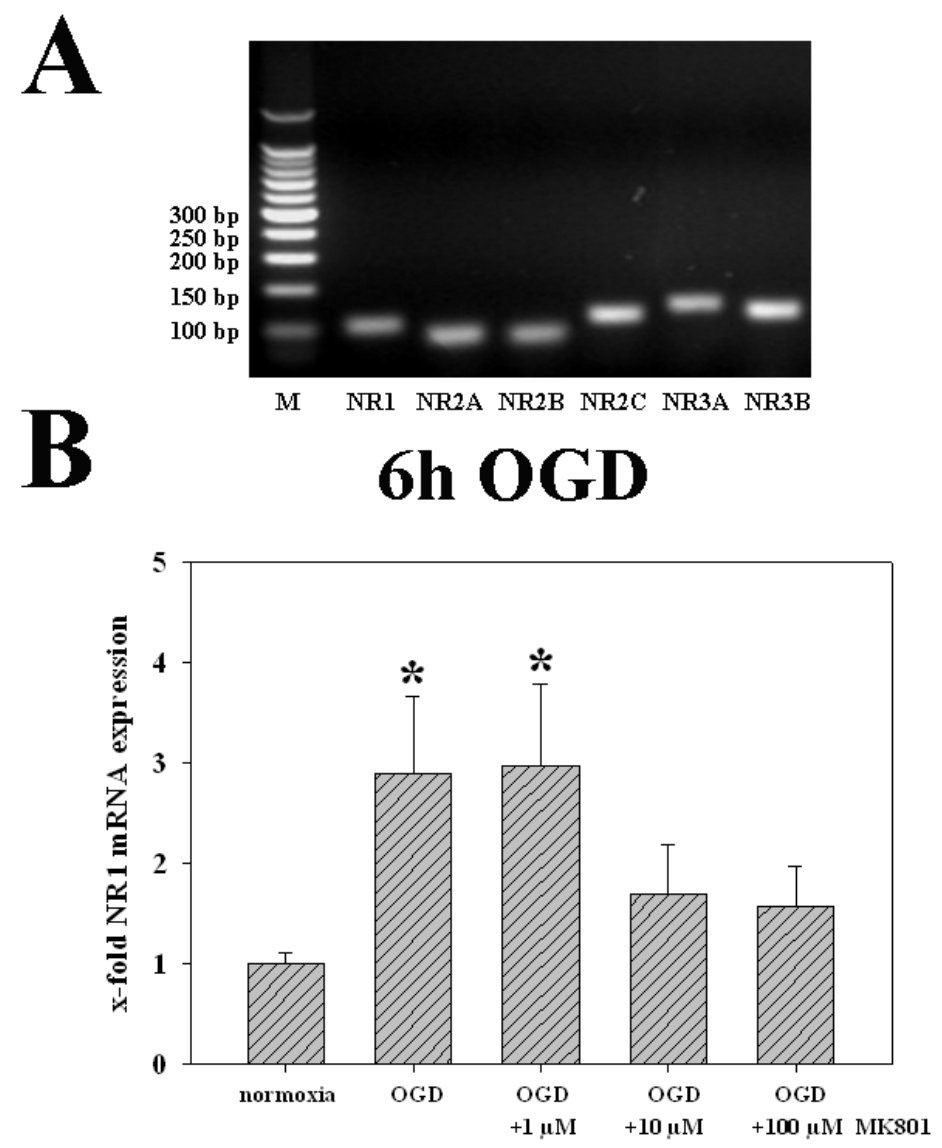

6h OGD + 20h RO

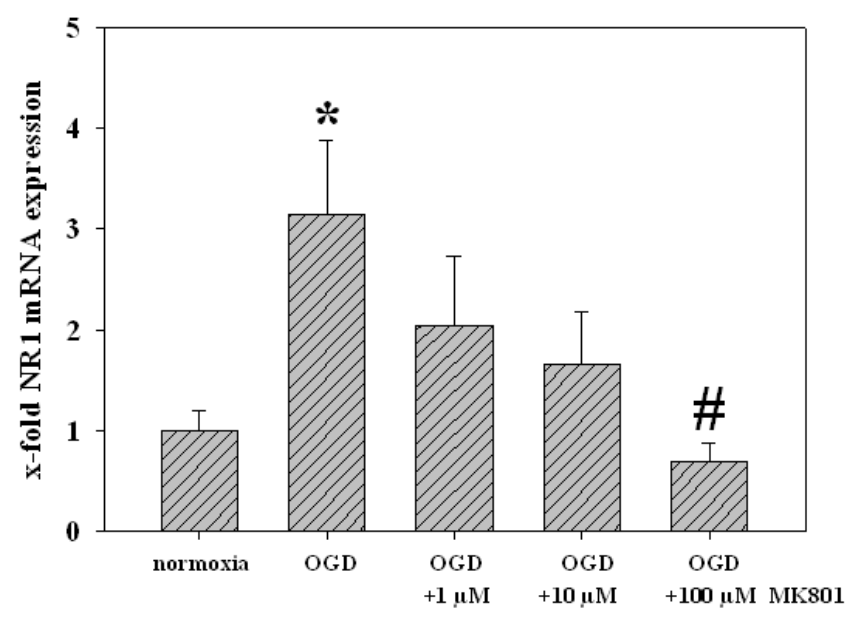




\section{Figure 4:}

\section{6h OGD}

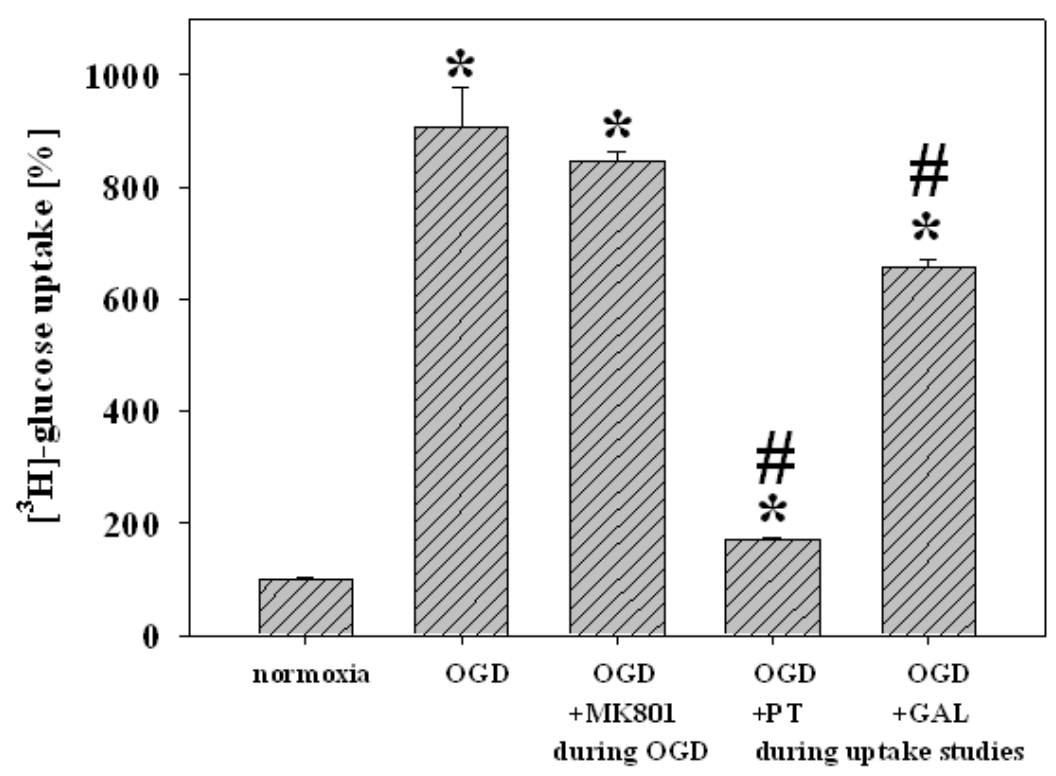

6h OGD + 20h RO

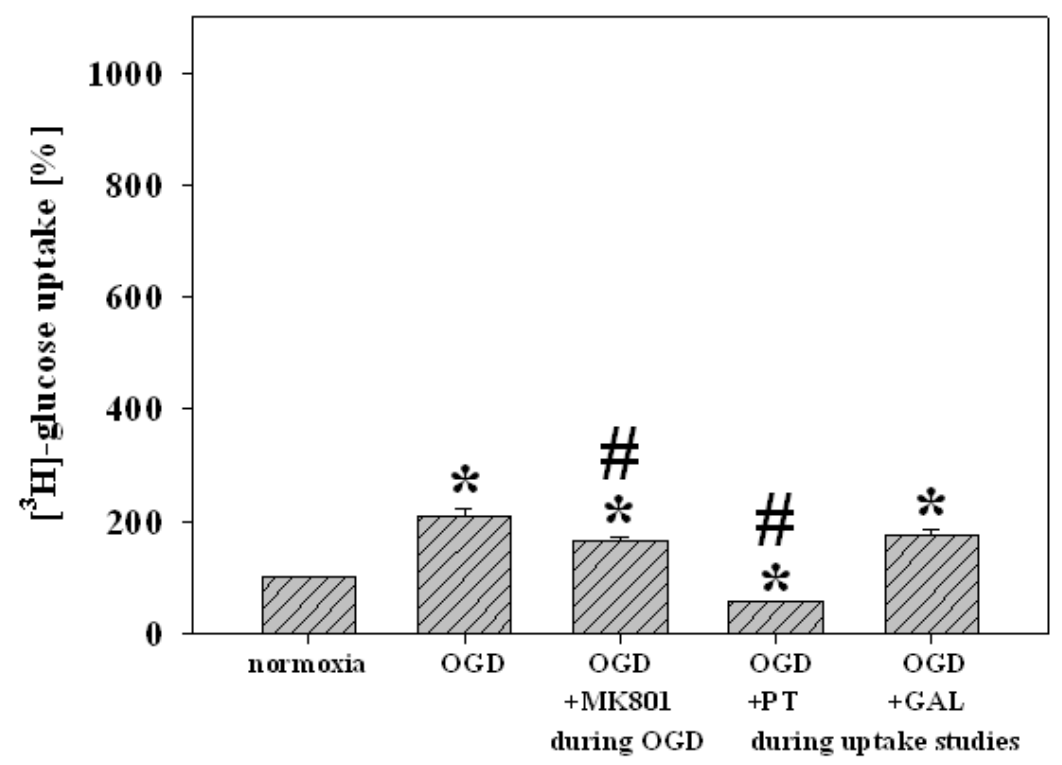

